\title{
非化学计量比调控高电压尖晶石正极材料电化学性能研究
}

\author{
赛喜雅勒图 $^{1,2}$, 王雪莹 ${ }^{2}$, 顾庆文 ${ }^{2}$, 夏永高 ${ }^{2}$, 刘兆平 ${ }^{2}$, 何 杰 $^{1}$
}

(1. 安徽理工大学 化学工程学院, 淮南 232001; 2. 中国科学院 宁波材料技术与工程研究所, 宁波 315201)

摘 要: 用一种简单的方法制备了高性能的高电压尖晶石正极材料, 主要是调控正极材料中锂与过渡金属的摩尔比, 即通过 $\mathrm{Ni}_{0.25} \mathrm{Mn}_{0.75}(\mathrm{OH})_{2}$ 与 $\mathrm{Li}_{2} \mathrm{CO}_{3}$ 进行高温固相反应制备了非化学计量比的 $\mathrm{Li}_{1.05} \mathrm{Ni}_{0.5} \mathrm{Mn}_{1.5} \mathrm{O}_{4}$ 和化学计量比的 $\mathrm{LiNi}_{0.5} \mathrm{Mn}_{1.5} \mathrm{O}_{4}$ 尖晶石型高电压正极材料。用扫描电子显微镜、 $\mathrm{X}$ 射线衍射、中子衍射、拉曼光谱、 $\mathrm{X}$ 射线光电子 能谱以及循环伏安曲线对其形貌、晶体结构及元素价态和电化学性能进行了表征。研究发现, 非化学计量比的 $\mathrm{Li}_{1.05} \mathrm{Ni}_{0.5} \mathrm{Mn}_{1.5} \mathrm{O}_{4}$ 中由于金属离子随机分布于 $16 \mathrm{~d}$ 位置, 所以 $\mathrm{Ni} / \mathrm{Mn}$ 阳离子无序化程度更高。非化学计量比的高电 压正极材料具有更为优异的倍率性能, 并且在 400 次循环后比容量保持率高达 $91.2 \%$ 。同时, 原位 X 射线衍射测试 结果表明, 在充放电过程中非化学计量比的高电压正极材料发生连续单一的相转变, 可以提高晶体结构的稳定性。 因此，非计量比的尖晶石 $\mathrm{Li}_{1.05} \mathrm{Ni}_{0.5} \mathrm{Mn}_{1.5} \mathrm{O}_{4}$ 正极材料在高能量密度的锂离子电池中具有更广阔的应用前景。

关 键 词: 高电压; 尖晶石; 正极材料; 非化学计量比; 循环稳定性

中图分类号: TQ174 文献标识码: A

\section{Tuning Electrochemical Performance through Non-stoichiometric Compositions in High-voltage Spinel Cathode Materials}

\author{
LEE Sai-Xi ${ }^{1,2}$, WANG Xue-Yin ${ }^{2}$, GU Qing-Wen ${ }^{2}$, XIA Yong-Gao ${ }^{2}$, LIU Zhao-Ping ${ }^{2}$, HE Jie $^{1}$ \\ (1. School of Chemical Engineering, Anhui University of Science and Technology, Huainan 232001, China; 2. Ningbo Institute \\ of Materials Technology \& Engineering, Chinese Academy of Sciences, Ningbo 315201, China)
}

\begin{abstract}
In this study, a simple method to prepare high-voltage spinel cathode materials though controlling stoichiometric ratio in their compositions was reported. Non-stoichiometric and stoichiometric high-voltage spinel $\mathrm{LiNi}_{0.5} \mathrm{Mn}_{1.5} \mathrm{O}_{4}$ cathode materials were prepared by solid-state reaction between $\mathrm{Li}_{2} \mathrm{CO}_{3}$ and $\mathrm{Ni}_{0.25} \mathrm{Mn}_{0.75}(\mathrm{OH})_{2}$ prcursor. Their morphologies, structures and electrochemical performance were characterized by scanning electron microscopy, X-ray diffraction, neutron diffraction, Raman spectroscopy, X-ray photoelectron spectroscopy, as well as electrochemical curves. The second particles occupied the similar sizes $\sim 8 \mu \mathrm{m}$, which were composed of nanoparticles. Compared to stoichiometric $\mathrm{LiNi}_{0.5} \mathrm{Mn}_{1.5} \mathrm{O}_{4}, \mathrm{Ni} / \mathrm{Mn}$ cations in non-stoichiometric $\mathrm{LiNi}_{0.5} \mathrm{Mn}_{1.5} \mathrm{O}_{4}$ sample distributed randomly, resulting in structure disorder demonstrated by the analysis of X-ray diffraction, neutron diffration and Raman spectroscopy. Less $\mathrm{Mn}^{3+}$ content in stoichiometric $\mathrm{LiNi}_{0.5} \mathrm{Mn}_{1.5} \mathrm{O}_{4}$ sample was detected though X-ray photoelectron spectroscopy. It is believed that more $\mathrm{Mn}^{3+}$ content and $\mathrm{Ni} / \mathrm{Mn}$ cation disorder would benefit rate cpability and cycling performance. As a result, non-stoichiometric $\mathrm{LiNi}_{0.5} \mathrm{Mn}_{1.5} \mathrm{O}_{4}$ sample delivers superior dicharge capacity at higher rates, even though it shows relatively minor discharge capacity at low rates. What's more, higher capacity retention for the non-stoichiometric $\mathrm{LiNi}_{0.5} \mathrm{Mn}_{1.5} \mathrm{O}_{4}$ sample was found, which was promoted to $91.2 \%$ at $1.0 \mathrm{C}$ rate after 400 cycles. At the same time, in situ X-ray diffraction measurements revealed that single-step
\end{abstract}


phase transformation for non-stoichiometric $\mathrm{LiNi}_{0.5} \mathrm{Mn}_{1.5} \mathrm{O}_{4}$ sample significantly enhanced structural stability during the electrochemical process. Spinel $\mathrm{LiNi}_{0.5} \mathrm{Mn}_{1.5} \mathrm{O}_{4}$ with non-stoichiometric composition provides a promising solution for their potential application in high-energy-density lithium-ion batteries.

Key words: high voltage; spinel-structured; cathode materials; non-stoichiometric; cycling stability

近年来, 锂离子动力电池在纯电动汽车和混合 动力汽车领域得到了广泛的应用, 但是成本及续航 里程问题一直是锂离子动力电池迫切需要解决的问 题 ${ }^{[1]}$ 。锂离子动力电池对高能量密度和高输出功率 的需求, 使得高电压正极材料的研发受到了人们的 广泛关注。 $\mathrm{LiNi}_{0.5} \mathrm{Mn}_{1.5} \mathrm{O}_{4}(\mathrm{LNMO})$ 材料晶体结构稳 定, 可以提供三维锂离子传输通道, 具有 $4.7 \mathrm{~V}(\mathrm{vs}$. $\left.\mathrm{Li}^{+} / \mathrm{Li}^{0}\right)$ 高电压平台, 理论比容量达到 $147 \mathrm{mAh} / \mathrm{g}$, 且其拥有较好的倍率性能和循环稳定性 ${ }^{[2-6]}$ 。同时, 与已经商业化的正极材料 $\mathrm{LiMn}_{2} \mathrm{O}_{4}(\sim 400 \mathrm{Wh} / \mathrm{kg})$ 、 $\mathrm{LiFePO}_{4}(\sim 495 \mathrm{Wh} / \mathrm{kg}) 、 \mathrm{LiNi}_{1 / 3} \mathrm{Co}_{1 / 3} \mathrm{Mn}_{1 / 3} \mathrm{O}_{2}(\sim 576 \mathrm{Wh} / \mathrm{kg})$ 相比较 ${ }^{[7]}, \mathrm{LiNi}_{0.5} \mathrm{Mn}_{1.5} \mathrm{O}_{4}$ 材料的能量密度高达 $650 \mathrm{Wh} / \mathrm{kg}$, 更加适用于混合动力和电动汽车用锂离子电池。但 是在循环过程中, $\mathrm{LiNi}_{0.5} \mathrm{Mn}_{1.5} \mathrm{O}_{4}$ 电极材料在高电压 下其材料颗粒表面结构发生改变, 从而引起材料表 面的物质从电极材料中溶解后进入电解液, 导致电 池库伦效率降低, 放电容量急剧衰减 ${ }^{[7-14]}$ 。

LNMO 通常是立方相尖晶石结构, 存在两种不 同结构的空间群: 一种是 Ni/Mn 无序的 Fd-3m 空间 群, $\mathrm{Mn}$ 元素的价态为 +3 和 +4 价, 材料中存在部分 氧缺陷; 另一种是 $\mathrm{Ni} / \mathrm{Mn}$ 有序 $\mathrm{P} 4332$ 空间群, $\mathrm{Mn}$ 元 素均为 +4 价, 材料中不存在氧缺陷 ${ }^{[15]}$, 这两种结构 在一定的合成条件下可以相互转变 ${ }^{[7,16]}$ 。有研究认 为, 因为 $\mathrm{Fd}-3 \mathrm{~m}$ 结构锂离子扩散系数较高, 所以 Fd-3m 型材料比 $\mathrm{P} 4332$ 型材料具有更好的倍率性能。 其主要原因是 Fd-3m 结构正极材料在充放电过程中 仅进行单一相转变 ${ }^{[17-18]}$, 而 $\mathrm{P} 4332$ 结构材料在充放 电过程中还会出现一种类似 Fd-3m 结构的中间相, 需经过两步相转变过程, 所以 Fd-3m 结构的循环性 能更优。而两者最为显著的区别是在 Fd-3m 材料中 含有微量的 $\mathrm{Mn}^{3+}$ 元素, 而在 $\mathrm{P}_{3} 32$ 材料中 $\mathrm{Mn}$ 的化 合价完全为 +4 价。因此, 研究者尝试通过各种方法 来实现高电压正极材料从 $\mathrm{P}_{3} 32$ 结构转变到 $\mathrm{Fd}-3 \mathrm{~m}$ 结构, 主要包括表面改性 ${ }^{[19]}$ 元素掺杂 ${ }^{[15,20]}$ 等。Xiao 等 ${ }^{[15]}$ 通过掺杂部分 $\mathrm{Cr}^{3+}$ 来调控 $\mathrm{Mn}^{3+}$ 离子的浓度, 来 改变其结构的有序化程度, 改善其电化学性能。Jo 等 ${ }^{[19]}$ 在高电压正极材料表面进行磷酸盐化处理, 加 剧其结构的无序化程度, 从而显著提高倍率和循环 性。最近, Lee 等 ${ }^{[20]}$ 利用中子衍射等表征手段发现, 掺杂少量 $\mathrm{Li}$ 离子到 $\mathrm{Ni}$ 的位置可以改变 $\mathrm{Ni} / \mathrm{Mn}$ 的无
序化程度, 而对电化学性能没有产生任何影响。这 些研究结果表明, 在高电压尖晶石材料中, 不同缺 陷浓度的存在对于其电化学性能具有重要的作用。 然而, 在这些研究中合成制备过程过于复杂, 所以 在优化条件降低材料制备成本的同时，兼顾优异的 电化学性能制备高性能的正极材料具有更重要的现 实意义。

本研究通过高温固相法制备了非化学计量比和 化学计量比的 LNMO 正极材料。采用扫描电子显微 镜、粉末 $X$ 射线衍射、中子衍射、拉曼光谱、 $X$ 射 线光电子能谱研究了微观形貌和微观结构的变化, 以及采用原位的 XRD 研究了非化学计量比和化学计 量比的正极材料晶体结构的演化过程, 最后建立了材 料的微观结构与电化学性能之间的关系。

\section{1 实验方法}

\section{1 样品制备}

在氮气保护下, 将浓度为 $2 \mathrm{~mol} / \mathrm{L}$ 的 $\mathrm{NiSO}_{4}$ 和 $\mathrm{MnSO}_{4}$ 的过渡金属混合溶液(摩尔比为 $\mathrm{Ni}: \mathrm{Mn}=1: 3$ ) 加入连续摚拌反应釜中, 同时将 $5 \mathrm{~mol} / \mathrm{L}$ 的 $\mathrm{NaOH}$ 溶 液和作为络合剂的一定量 $\mathrm{NH}_{4} \mathrm{OH}$ 分别加到反应釜 中, 将反应釜中 $\mathrm{pH}$ 控制在 11.5 、温度 $60^{\circ} \mathrm{C}$ 和搅拌 速度 $800 \mathrm{r} / \mathrm{min}$, 把沉淀物洗涤、烘干后得到球形 $\mathrm{Ni}_{0.25} \mathrm{Mn}_{0.75}(\mathrm{OH})_{2}$ 。

以 $\mathrm{Ni}_{0.25} \mathrm{Mn}_{0.75}(\mathrm{OH})_{2}$ 和 $\mathrm{Li}_{2} \mathrm{CO}_{3}(>99.5 \%$, 质量分 数)为原料, 按照摩尔比 $1: 0.2625$ 称量, 放到玛瑙 研钵中研磨, 再将混合均匀的物料放入马弗炉中, 以 $5^{\circ} \mathrm{C} / \mathrm{min}$ 的升温速度升至 $900^{\circ} \mathrm{C}$, 焙烧 $16 \mathrm{~h}$ 后自 然冷却至室温, 得到一次烧结后的产物。然后将一 次烧结后的产物放入马弗炉中, 以 $5^{\circ} \mathrm{C} / \mathrm{min}$ 的升温 速度升至 $700^{\circ} \mathrm{C}$, 焙烧 $8 \mathrm{~h}$, 自然冷却至室温, 得到 $\mathrm{Li}_{1.05} \mathrm{Ni}_{0.5} \mathrm{Mn}_{1.5} \mathrm{O}_{4}$ 正极材料, 标记为 Non-stoichiometricLNMO。作为对比样品, 以 $\mathrm{Ni}_{0.25} \mathrm{Mn}_{0.75}(\mathrm{OH})_{2}$ 和 $\mathrm{Li}_{2} \mathrm{CO}_{3}$ 为原料, 按照摩尔比 $1: 0.25$ 称量, 放入玛瑙研钵中 研磨，在同样条件下反应得到 $\mathrm{LiNi}_{0.5} \mathrm{Mn}_{1.5} \mathrm{O}_{4}$ 正极材 料, 标记为 Stoichiometric- LNMO。

\section{2 结构表征}

采用 FEI QUANTA 250 FEG 型场发射扫描电子 显微镜(美国 FEI 公司)测试高电压正极材料的微观 
形貌。采用 D8 Discover 型 X 射线衍射仪(德国布鲁 克公司)测试高电压正极材料的结构(采用 $\mathrm{Cu} \mathrm{K \alpha}$, $\lambda=0.1541 \mathrm{~nm}$, 管压 $40.0 \mathrm{kV}$, 管电流 $40 \mathrm{~mA}$, 扫描速 率 $6^{\circ} / \mathrm{min}$, 扫描范围 $10^{\circ} \sim 70^{\circ}$ )。采用 inVia-reflex 型 显微共聚拉曼光谱仪(雷尼绍公司)测试高电压正极 材料的光谱响应特征。通过 AXIS Ultra DLD 谱仪对 样品进行 $X$ 射线光电子谱(XPS)分析。采用单色 $\mathrm{Al}-\mathrm{K} \alpha \mathrm{X}$ 射线放射源 $(1486.6 \mathrm{eV})$ 收集谱图, 功率为 $150 \mathrm{~W}$, 能量为 $10 \mathrm{eV}$; 利用橡树岭国家实验室 (ORNL)的散列中子源的 VULCAN 站线, 采用飞行 时间的模式收集中子粉末衍射数据(ND)。将高电压 尖晶石正极材料粉末填充在一个钒样品器血中, 入 射波束 $(5 \mathrm{~mm} \times 12 \mathrm{~mm})$ 的带宽为 0.07 至 $0.35 \mathrm{~nm}$, 其 允许 $d$ 值在 $0.05 \sim 0.25 \mathrm{~nm}$ 内, 从而使衍射仪探测器 在 $2 \theta=-90^{\circ} \sim 90^{\circ}$ 内移动, 利用频率为 $30 \mathrm{~Hz}$ 的双磁 盘升降仪在粉末持续照射时间为 $3 \mathrm{~h}$ 的高分辨率模 式下采集中子粉末衍射数据, 并通过 VDRIVE 软 件校正。

\section{3 电化学性能测试}

以 $N$-甲基吡咯烷酮(NMP)为溶剂，高电压尖晶 石正极材料、乙炔黑、聚四氟乙烯(PVDF)按质量比 $90: 5: 5$ 混合均匀制成浆料, 涂覆在铝箔上, $120^{\circ} \mathrm{C}$ 真空干燥 $12 \mathrm{~h}$ 。以 Celgard2400 聚丙烯多孔膜为隔 膜, $1 \mathrm{~mol} / \mathrm{L} \mathrm{LiPF}_{6}$ 的碳酸乙烯酯 $(\mathrm{EC})$ 和碳酸二甲酯 $(\mathrm{DMC})$ 混合溶液 $(\mathrm{EC}: \mathrm{DMC}=1: 1)$ 为电解液, 以金属 锂片为负极, 在充满氩气的手套箱内组装成扣式电 池 $\left(\mathrm{H}_{2} \mathrm{O}<0.1 \times 10^{-6}, \mathrm{O}_{2}<0.1 \times 10^{-6}\right)$ 。将组装好的扣式电 池用 LAND 电池测试系统(武汉金诺电子有限公司) 对扣式电池进行常温循环性能测试。通过输力强电 化学工作站对样品进行循环伏安测试, 测试电压范 围为 $3.0 \sim 5.0 \mathrm{~V}$, 扫描速率为 $0.2 \mathrm{mV} / \mathrm{s}$ 。

\section{4 原位 XRD 测试}

原位电池的组装按照 1.3 实验部分电池组装来 实施。将装好的模拟电池用 LAND 电池测试系统(武 汉金诺电子有限公司)进行 $0.1 C$ 充放电循环，同时用 D8 Discover 型 X 射线衍射仪(德国布鲁克公司)对模 拟电池正极壳的铍窗位置进行测试并采集数据，就 可以得到高电压正极材料在不同充电(SOC)和放电 (DOD)深度时的晶体结构(采用 $\mathrm{Cu} \mathrm{K} \alpha, \lambda=0.1541 \mathrm{~nm}$, 管压 $40.0 \mathrm{kV}$, 管电流 $40 \mathrm{~mA}$, 扫描速率 $6 \% \mathrm{~min}$, 扫 描范围 $10^{\circ} \sim 70^{\circ}$ )。

\section{2 结果与讨论}

\section{1 微观形貌和相结构分析}

图 1 为 Non-stoichiometric-LNMO 和 Stoichiometric-
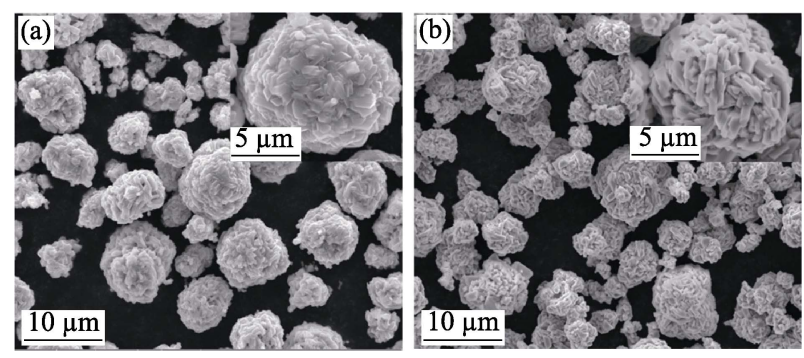

图 1 (a) Non-stoichiometric-LNMO 和(b)Stoichiometric-LNMO 样品的 SEM 照片

Fig. 1 SEM images of (a) Non-stoichiometric-LNMO and (b) Stoichiometric-LNMO

LNMO 样品的 SEM 照片, 从图中可见，两样品均呈 球形形貌, 球形颗粒的平均尺寸为 $\sim \mu \mathrm{m}$; 球形颗 粒的形貌为一次颗粒组成的二次颗粒。此微观形貌 结构一方面有利于提高材料的振实密度, 另一方面 有利于缩短锂离子的传输路径 ${ }^{[21]}$ 。此外, 两者的一 次颗粒大小没有显著的区别, 但是由一次颗粒组成 的二次颗粒明显不同, 非化学计量比样品的材料结 构明显密实，这种密实结构更利于提高材料的循环 稳定性。

图 2 为 Non-stoichiometric-LNMO 和 StoichiometricLNMO 样品的 X 射线精修衍射图谱, Non-stoichiometricLNMO 样品采用 Fd-3m 结构模型, StoichiometricLNMO 样品采用 $\mathrm{P}_{3} 32$ 结构模型。从图中可以
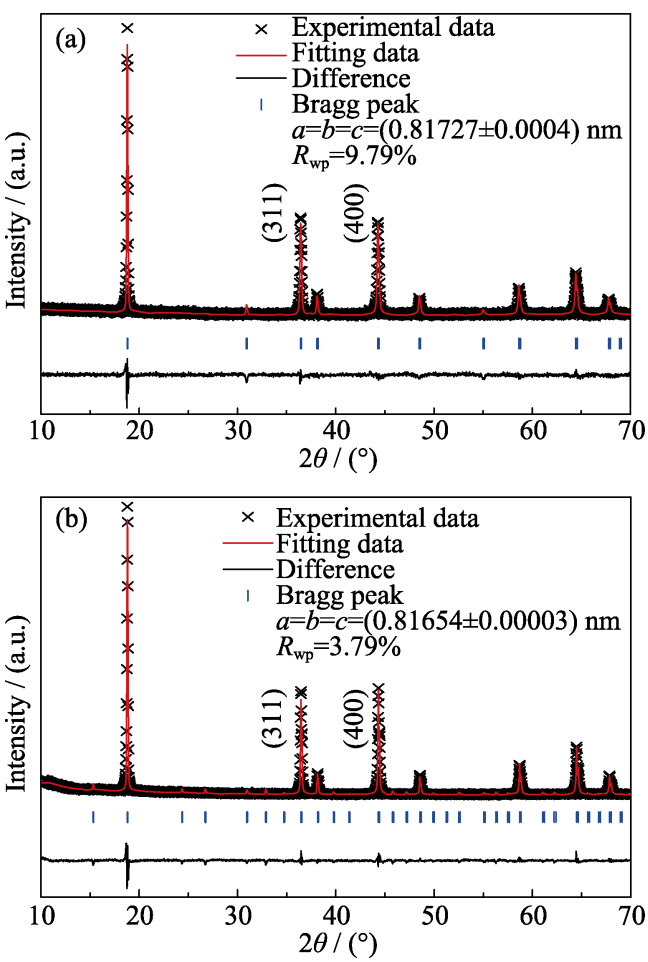

图 2 (a) Non-stoichiometric-LNMO 和(b)Stoichiometric-LNMO 样品的 XRD 图谱

Fig. 2 XRD patterns of (a) Non-stoichiometric-LNMO and (b) Stoichiometric-LNMO with refinement results 
看出, 两个样品均具有较高的结晶度。通常利用 (311)和(400)峰强度的比值来判定材料中阳离子的 混排度 $(\gamma), \gamma$ 表示材料中 $8 \mathrm{a}$ 位置的 $\mathrm{Li}$ 离子和 $16 \mathrm{~d}$ 位 置的 $\mathrm{Mn}$ 或 $\mathrm{Ni}$ 离子的混排程度。 $\gamma$ 越高, $I_{(311)} / I_{(400)}$ 也越大 ${ }^{[22]}$ 。 Non-stoichiometric-LNMO 样品的 $I_{(311)} / I_{(400)}$ 为 1.0364 , 明显高于 Stoichiometric-LNMO 样 品(0.9758), 这说明在 Non-stoichiometric-LNMO样品 中阳离子的混排程度高于 Stoichiometric-LNMO 样 品。同时, 过量的 Li 离子导致 Non-stoichiometricLNMO 样品的晶胞参数 $a=0.81727 \mathrm{~nm}$ 略微增大 $(a=0.81654 \mathrm{~nm})$, 其主要原因可能是由于过量的 $\mathrm{Li}$ 离子导致其 $\mathrm{Mn}$ 的化合价态从 +4 价转变成 +3 价(电 化学曲线分析)。此外, 由于 $\mathrm{X}$ 射线对于 $\mathrm{Ni}$ 和 $\mathrm{Mn}$ 具有相近的散射长度, 导致无法观察到 $\mathrm{Ni} / \mathrm{Mn}$ 阳离 子之间的混排程度。

中子衍射被普遍认为是一种辨别不同过渡金属 元素位置理想的工具, 可以利用其进一步探讨非化 学计量比组成对 $\mathrm{Ni} / \mathrm{Mn}$ 阳离子混排程度的影响。 $\mathrm{Ni}$ 和 $\mathrm{Mn}$ 对于中子具有不同的相干散射长度 ${ }^{[23]}$, 如 $\mathrm{Ni}$ 的散射长度为 $10.3 \mathrm{fm}$, 而 $\mathrm{Mn}$ 的散射长度为 $-3.73 \mathrm{fm}$ 。 图 3 为实验样品的中子衍射图谱。由图可见, 相比 较于 Stoichiometric-LNMO 样品, Non-stoichiometricLNMO 样品具有更多阳离子无序化的特点, 即 $\mathrm{Ni} / \mathrm{Mn}$ 阳离子无序化程度加剧, 其结构从有序化的
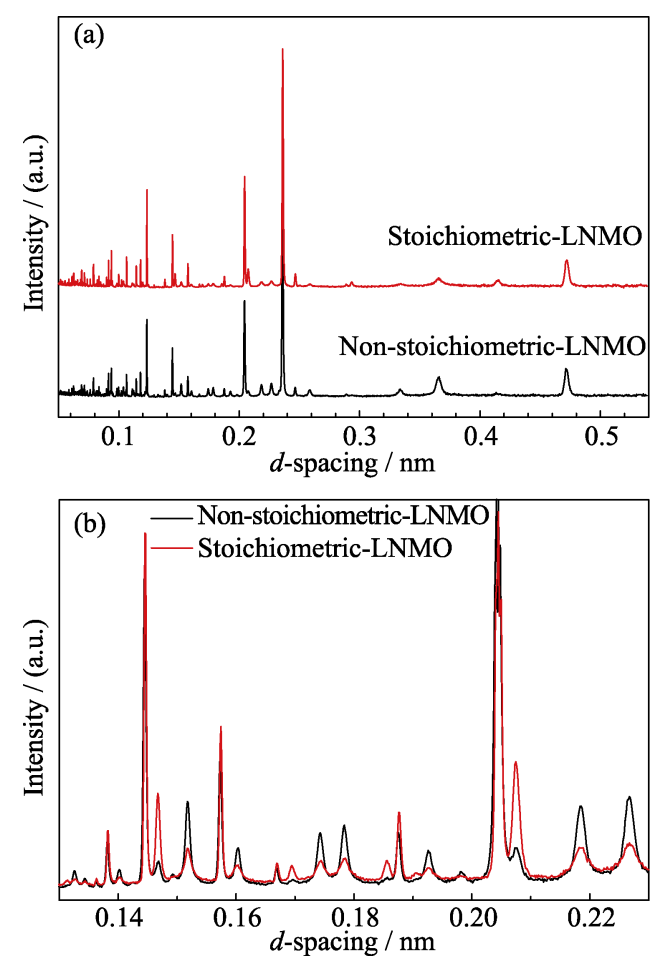

图 3 (a) Non-stoichiometric-LNMO 和 (b) StoichiometricLNMO 样品的中子衍射图谱

Fig. 3 Neutron diffraction patterns of (a) Non-stoichiometricLNMO and (b) Stoichiometric-LNMO
$\mathrm{P} 4{ }_{3} 32$ 结构转变成无序化的 $\mathrm{Fd}-3 \mathrm{~m}$ 结构 ${ }^{[22]}$ 。

对于 $\mathrm{Ni} / \mathrm{Mn}$ 阳离子的有序性程度导致的晶体对 称性变化, 拉曼光谱是一个有力的考察手段。图 4 为实验样品的拉曼光谱。图中 $638.8 \mathrm{~cm}^{-1}$ 处的 $\mathrm{A}_{1 \mathrm{~g}}$ 峰以及在 594.7 和 $611.3 \mathrm{~cm}^{-1}$ 的 $\mathrm{F}_{2 \mathrm{~g}(1)}$ 峰对应于 $\mathrm{Mn}-\mathrm{O}$ 键的对称性伸缩振动, 与此同时, 在 $408.4 \mathrm{~cm}^{-1}$ 处 的 $\mathrm{E}_{\mathrm{g}}$ 峰和 $498.4 \mathrm{~cm}^{-1}$ 处的 $\mathrm{F}_{2 \mathrm{~g}(2)}$ 峰对应于 $\mathrm{Ni}-\mathrm{O}$ 键的 伸缩振动。相比于 Stoichiometric-LNMO 样品, Non-stoichiometric-LNMO 样品在 638.8、498.4 和 $408.4 \mathrm{~cm}^{-1}$ 处峰的强度显著降低, 这是由于 $\mathrm{Li}^{+}$掺杂 导致 $\mathrm{Ni} / \mathrm{Mn}$ 阳离子的有序化程度降低。此外, Non-stoichiometric-LNMO 样品中 $594.7 \mathrm{~cm}^{-1}$ 处的 $\mathrm{F}_{2 \mathrm{~g}(1)}$ 峰存在着更明显的䢃裂, 这种现象主要是由于 $\mathrm{Ni} / \mathrm{Mn}$ 的无序化程度增加导致其结构从有序的 $\mathrm{P}_{3} 32$ 结构转变成无序的 Fd-3m 结构 ${ }^{[24-25]}$ 。结果进 一步说明在 Non-stoichiometric-LNMO 样品中 $\mathrm{Ni} / \mathrm{Mn}$ 阳离子无序化程度更高, 而这种结构对于其 电化学性能具有重要的影响。

\section{$2.2 \mathrm{X}$ 射线光电子能谱分析}

采用 X 射线光电子能谱考察 Non-stoichiometricLNMO 和 Stoichiometric-LNMO 样品表面 $\mathrm{Mn}^{3+}$ 和 $\mathrm{Mn}^{4+}$ 浓度的差别, 结果如图 5 所示。从图中可以看 出, 无论 Non-stoichiometric-LNMO 还是 StoichiometricLNMO, 材料中 $\mathrm{Mn}^{3+}$ 和 $\mathrm{Mn}^{4+}$ 浓度都基本相同。此 外, 通过比较两者的积分面积发现, Non-stoichiometricLNMO 样品的 $\mathrm{Mn}^{3+}$ 浓度更高, 这说明 StoichiometricLNMO 中同样含有 $\mathrm{Mn}^{3+}$ 离子, 只是浓度低于 Nonstoichiometric-LNMO。

\section{3 电化学性能}

图 6(a)为 Non-stoichiometric-LNMO 和 Stoichiometric-LNMO 样品在电流密度为 $0.2 C$ 条件下(测试 电压范围: 3.0 4.95 V)的首次充放电曲线图。结果显 示, Non-stoichiometric-LNMO 样品的首次放电比容



图 4 Non-stoichiometric-LNMO 和 Stoichiometric-LNMO 样 品的拉曼光谱

Fig. 4 Raman spectra of Non-stoichiometric-LNMO and Stoichiometric LNMO 

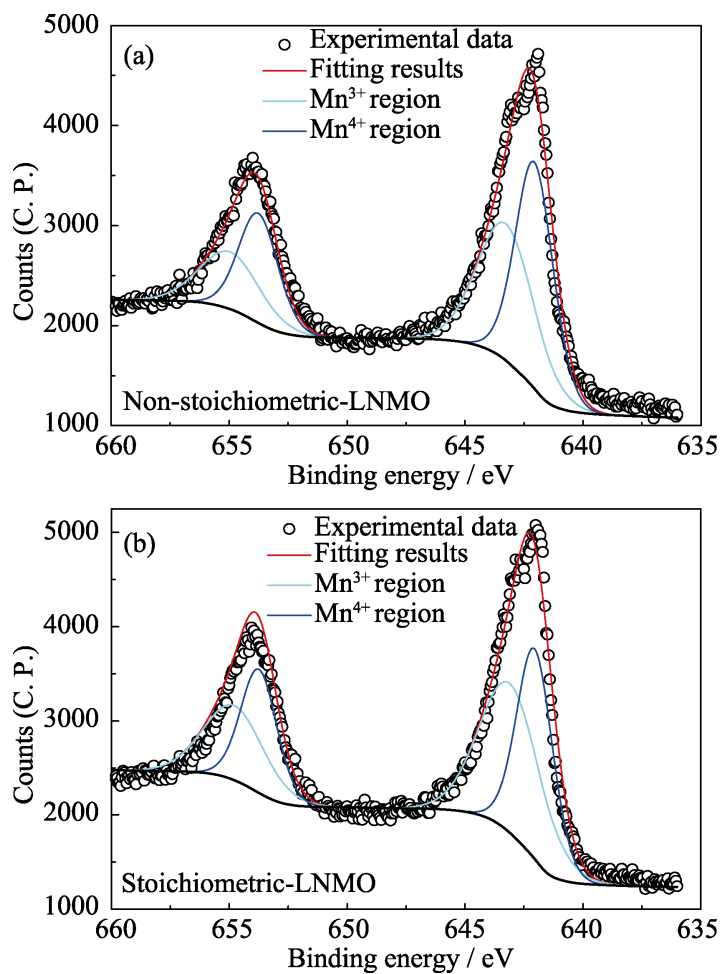

图 5 (a) Non-stoichiometric-LNMO 和(b) StoichiomericLNMO 样品的 Mn 2P 电子 X 射线光电子能谱图(XPS)

Fig. 5 XPS images of Mn 2P of (a) Non-stoichiometricLNMO and (b) Stoichiometric-LNMO

量为 $132.8 \mathrm{mAh} / \mathrm{g}$, 首次库伦效率为 $90.2 \%$, 而 Stoichiometric-LNMO 样品的首次放电比容量为 $136.8 \mathrm{mAh} / \mathrm{g}$, 首次库伦效率为 $81.6 \%$ 。也就是说非 化学计量比的材料能够显著提高材料的首次库伦效 率, 从而减少首次过程中锂离子的不可逆损失。此 外, 相比于 Stoichiometric-LNMO 样品, Non- stoichiometric-LNMO 样品的首次充放电曲线在 $4.0 \mathrm{~V}$ 有 较小的平台出现, 其对应于 $\mathrm{Mn}^{4+} / \mathrm{Mn}^{3+}$ 的氧化还原 过程 ${ }^{[26]}$ 。这说明加入过量 Li 离子导致 Non- stoichiometric-LNMO 样品中存在一定量的 $\mathrm{Mn}^{3+}$, 根据 上述结构表征结果得知该样品以无序化的 Fd-3m 结 构为主。而 Stoichiometric-LNMO 样品并没有在 4.0 $\mathrm{V}$ 出现平台, 说明结构中 $\mathrm{Mn}^{3+}$ 离子含量很低, 根据 上述结构表征结果得知该样品以有序化的 $\mathrm{P} 4332$ 结 构为主。

图 6(b)为 Non-stoichiometric-LNMO 和
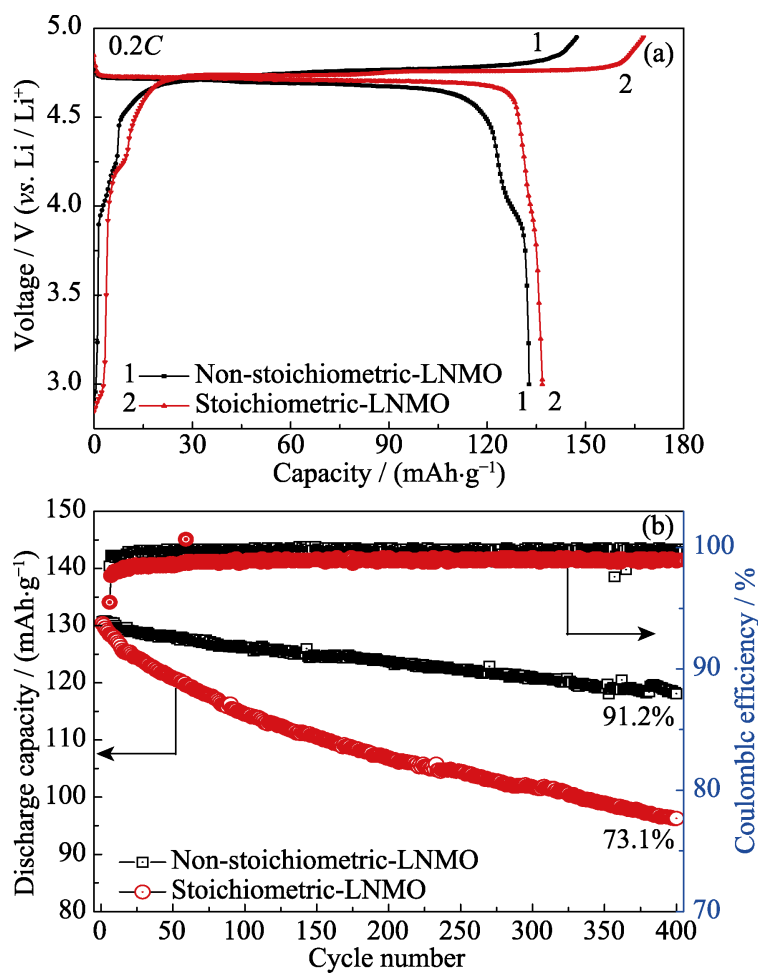

图 6 (a) Non-stoichiometric-LNMO 和 Stoichiometric-LNMO 样品在 $0.2 C$ 电流密度下的首次充放电曲线; (b) 在 $1.0 \mathrm{C}$ 电流 密度下的两个样品的循环性能以及库伦效率

Fig. 6 (a) Initial charge-discharge curves at $0.2 C$ rate, and (b) cycling performance as well as coulombic efficiency at $1.0 \mathrm{C}$ rate for Non-stoichiometric-LNMO and Stoichiometric-LNMO samples

Stoichiometric-LNMO 样品在电流密度为 $1.0 C$ 条件 下(测试电压范围: 3.0 4.95 V)循环性能和循环效率 图。从图中可见, Non-stoichiometric-LNMO 样品在 $1.0 C$ 首次放电比容量达到 $130 \mathrm{mAh} / \mathrm{g}$, 循环 400 次 后其放电比容量仍保有 $118.7 \mathrm{mAh} / \mathrm{g}$, 容量保持率 高达 91.2\%; 而 Stoichiometric-LNMO 样品经过 400 次循环后其放电比容量只有 $96.5 \mathrm{mAh} / \mathrm{g}$, 容量保持 率仅有 $73.1 \%$ 。此外, Non-stoichiometric-LNMO 样 品在循环过程中库伦效率明显高于 StoichiometricLNMO 样品。同时, 本研究的结果明显优于目前报 道的相关结果, 其具体内容如表 1 所示。从这些结 果可见，具有 $\mathrm{Ni} / \mathrm{Mn}$ 阳离子无序化结构能够显著提 高材料的循环稳定性。

表 1 不同合成方法制备的高压尖晶石材料性能的比较

Table 1 Comparison with different methods to prepare high-voltage spinel cathode materials

\begin{tabular}{cccc}
\hline Ref. & $1^{\text {st }}$ discharge capacity $/\left(\mathrm{mAh} \cdot \mathrm{g}^{-1}\right)$ & Cycling performance $/ \%$ & Rate capabiity $/\left(\mathrm{mAh} \cdot \mathrm{g}^{-1}\right)$ \\
\hline$[15]$ & $124(0.1 C)$ & $90(250$ cycles $)$ & $120(0.2 C$ charge, $5 C$ discharge $)$ \\
{$[19]$} & $129(0.1 C)$ & $83(300$ cycles $)$ & $110(1 C$ charge, $2 C$ discharge $)$ \\
{$[20]$} & $135(0.1 C)$ & $88(80$ cycles $)$ & - \\
This work & $132.8(0.2 C)$ & $91.2(400$ cycles $)$ & $122.4(1 C$ charge, $2 C$ discharge $)$ \\
\hline
\end{tabular}




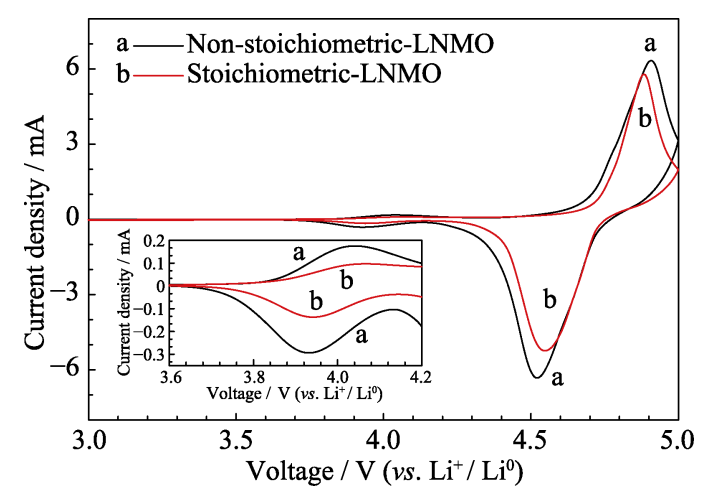

图 7 (a) Non-stoichiometric-LNMO 和 (b) Stoichiometric-LNMO 样品的循环伏安曲线图

Fig. $7 \mathrm{CV}$ curves of (a) Non-stoichiometric-LNMO and (b) Stoichiometric-LNMO at the scan rate of $0.2 \mathrm{mV} / \mathrm{s}$.

图 7 为 Non-stoichiometric-LNMO 和 Stoichiometric LNMO 样品的循环伏安 $(\mathrm{CV})$ 曲线。从 图中可以看出, Non-stoichiometric-LNMO 材料在电 压范围为 3.5 4.0 V 之间具有更强的氧化还原峰, 对 应于 $\mathrm{Mn}^{3+} / \mathrm{Mn}^{4+}$ 的氧化还原, 这说明 Non-stoichiometricLNMO 样品中 $\mathrm{Mn}^{3+}$ 离子更多, 这与 XPS 分析结果 相同。

图 8 为 Non-stoichiometric-LNMO 和 StoichiometricLNMO 样品的不同倍率充放电曲线。从图中可以看 出, Non-stoichiometric-LNMO 虽然在低倍率时其放 电比容量小于 Stoichiometric-LNMO, 但是倍率性能 明显更为优异，这说明具有 $\mathrm{Ni} / \mathrm{Mn}$ 阳离子无序化结 构能够显著提高材料的倍率性能。

\section{4 原位 $\mathrm{X}$ 射线衍射}

图 9 为 Non-stoichiometric-LNMO 和 StoichiometricLNMO 样品在不同充电(SOC)和放电(DOD)深度时 原位 $\mathrm{X}$ 射线衍射图谱，可以进一步探索无序化结构 对电化学过程中锂离子嵌入/脱出过程的影响。从图 9(a)中可以看出, 对于 Fd-3m 结构的 Non- stoichiometric-LNMO 样品，当充电到 30\%时，初始的 $\mathrm{Fd}-3 \mathrm{~m}$ 结构相含量递减, 并观察到 $\mathrm{Li}_{0.74} \mathrm{Ni}_{0.5} \mathrm{Mn}_{1.5} \mathrm{O}_{4}$ 的 $\mathrm{Fd}-3 \mathrm{~m}$ 立方相结构出现; 当充电到 $50 \%$ 时, 以另外 一种新 $\mathrm{Li}_{0.5} \mathrm{Ni}_{0.5} \mathrm{Mn}_{1.5} \mathrm{O}_{4}$ 的 $\mathrm{Fd}-3 \mathrm{~m}$ 立方相结构为主; 当充电大于 $50 \%$ 时, 随着 $\mathrm{Li}^{+}$离子的持续脱出, 出现 第三种 $\mathrm{Li}_{0.28} \mathrm{Ni}_{0.5} \mathrm{Mn}_{1.5} \mathrm{O}_{4}$ 的 $\mathrm{Fd}-3 \mathrm{~m}$ 立方相结构; 当 充电接近 $100 \%$ 时，最后一种 $\mathrm{Li}_{0.05} \mathrm{Ni}_{0.5} \mathrm{Mn}_{1.5} \mathrm{O}_{4}$ 的 Fd-3m 立方相结构占主导地位。由于 Nonstoichiometric-LNMO 样品在充电过程中, 锂离子从 四面体位 $8 \mathrm{a}$ 位移动至空的八面体 $16 \mathrm{c}$ 位 ${ }^{[27]}$, 所以 Non-stoichiometric-LNMO 样品从原始无序化的 $\mathrm{Fd}-3 \mathrm{~m}$ 结构逐步地连续转变成新的 $\mathrm{Li}_{0.05} \mathrm{Ni}_{0.5} \mathrm{Mn}_{1.5} \mathrm{O}_{4}$ Fd-3m 立方相结构, 而不存在其他中间结构。因此,
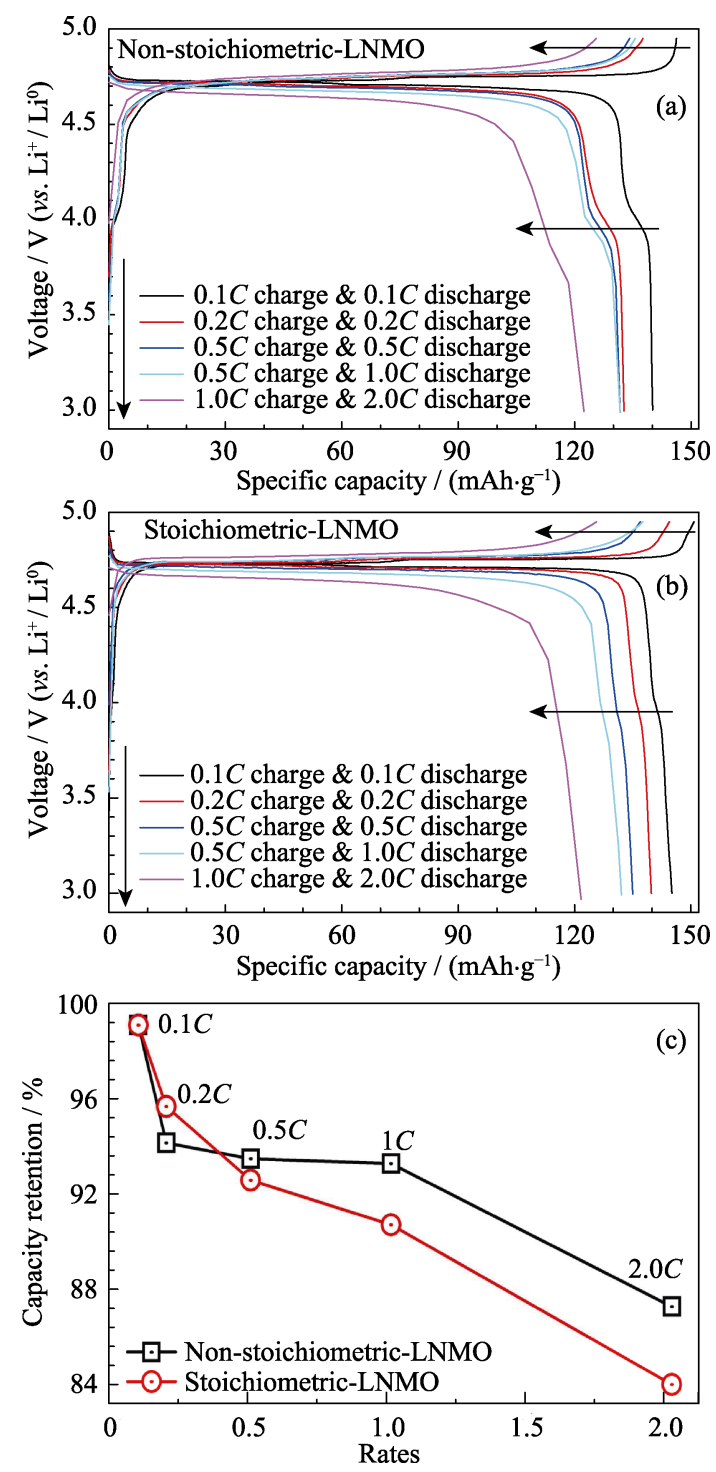

图 8 (a) Non-stoichiometric-LNMO, (b)StoichiometricLNMO 样品在不同倍率下的充放电曲线图和(c)倍率容量保 持率曲线图

Fig. 8 Charge-discharge curves of (a) Non-stoichiometricLNMO, (b) Stoichiometric-LNMO at different rates, and (c) Capacity retention at different rates

在原位 $\mathrm{X}$ 射线衍射图谱中，伴随着锂离子的脱出， 其变化过程是连续的, 促使 Non- stoichiometricLNMO 样品的(111)衍射峰连续向高角度方向偏移 (晶胞参数持续减小)。相比较而言, StoichiometricLNMO 样品在充电过程中, 由于空的八面体 $16 \mathrm{c}$ 位 被分为 $4 \mathrm{a}$ 位和 $12 \mathrm{~d}$ 位(比例为 $1: 3$ ), 锂离子从四面 体 $8 \mathrm{a}$ 位移动至八面体 $4 \mathrm{a}$ 位和 $12 \mathrm{~d}$ 位 ${ }^{[27]}$, 所以 Stoichiometric-LNMO 样品 X 射线衍射峰的位置发 生了三次比较明显的变化(图 9(b)), 说明存在不同 形式的相结构转变。Stoichiometric-LNMO 样品具有 更加有序的结构、在充放电过程中经历更大的应变, 需经过充电时的 $\mathrm{P}_{3} 32$ 相到 Fd-3m 相、放电时的 $\mathrm{Fd}-3 \mathrm{~m}$ 相到 $\mathrm{P} 4332$ 相两个步骤的相转变过程。由此 

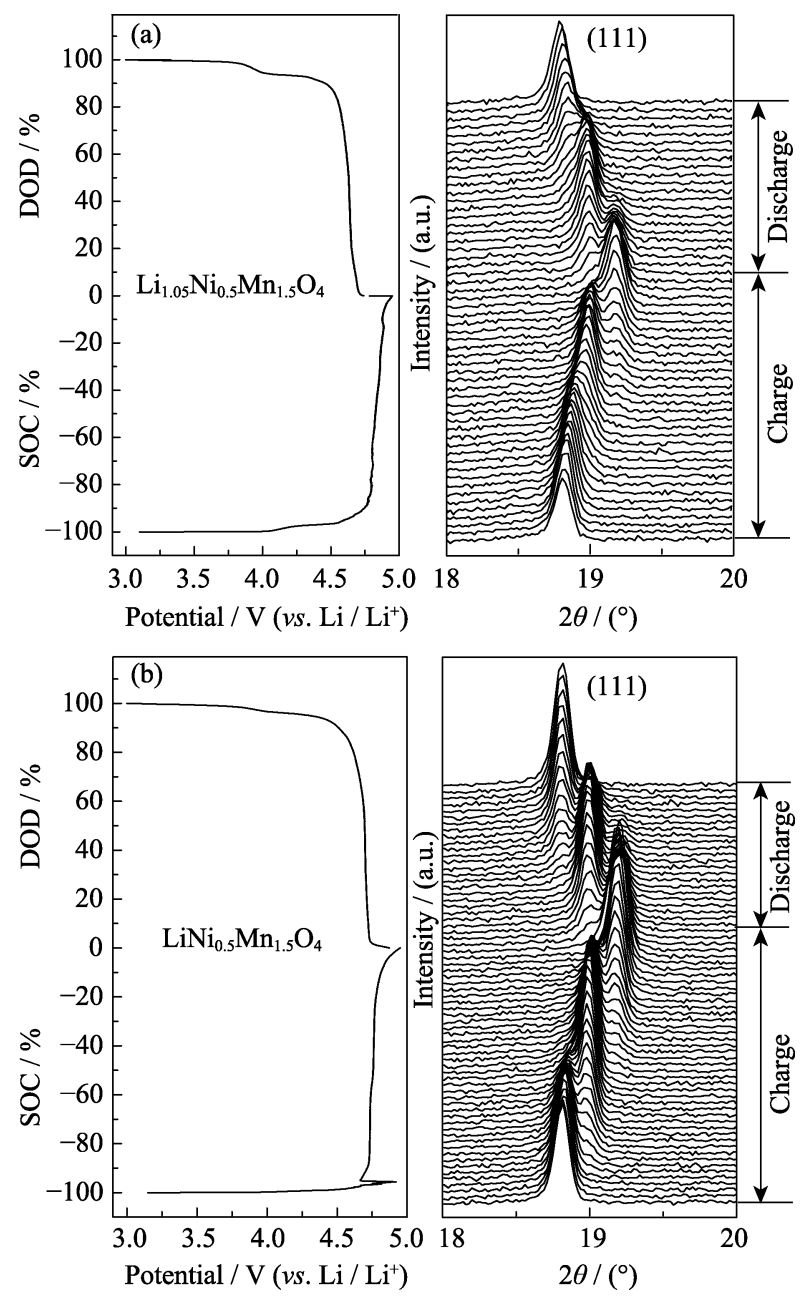

图 9 (a) Non-stoichiometric-LNMO 和(b) StoichiomericLNMO 的原位 XRD 图谱

Fig. 9 In situ XRD results during lithium-ion intercalation or deintercalation of (a) Non-stoichiometric-LNMO and (b) Stoichiometric-LNMO samples

可以得出, Non-stoichiometric-LNMO 样品在 $\mathrm{Li}^{+}$脱出/ 嵌入的过程中, 受到的晶格应变小于 StoichiometricLNMO 样品, 即 Non-stoichiometric-LNMO 样品具 有更为优异的晶体结构稳定性。

\section{3 结论}

本文采用调控正极材料中锂与过渡金属摩尔比 的方法制备了高性能的高电压尖晶石正极材料，该 材料具有非化学计量比的 Fd-3m 空间结构群。结果 表明, Ni/Mn 阳离子无序化程度更高的 Non- stoichiometric-LNMO 样品具有更为优异的晶体结构稳 定性，能够显著提高材料的循环稳定性。在 $25^{\circ} \mathrm{C}$ 温 度下，首次放电比容量 $132.8 \mathrm{mAh} / \mathrm{g}$ 、首次库伦效率 $90.2 \%$, 在 $1.0 C$ 倍率下放电比容量达到 $130 \mathrm{mAh} / \mathrm{g}$, 第 400 次循环放电比容量依然有 $118.7 \mathrm{mAh} / \mathrm{g}$, 容量保 持率达到 91.2\%。因此, 这种非计量比的高电压正
正极材料适用于高能量密度和高输出功率的动力锂 离子电池。

\section{参考文献:}

[1] BRUCE P G, FREUNBER S A, HARDWICK L J, et al. $\mathrm{Li}_{-}-\mathrm{O}_{2}$ and Li-S batteries with high energy storage. Nat. Mater., 2012, 11(10): 19-29.

[2] KANG B, CEDER G. Battery materials for ultra-fast charging and discharging. Nature, 2009, 458(7235): 190-193.

[3] MIZUSHIMA K, JONES P C P, WISEMAN J B, et al. $\mathrm{Li}_{x} \mathrm{CoO}_{2}$ $(0<x<1)$ : a new cathode material for batteries of high energy density. Mater. Res. Bull., 1980, 15(6): 783-789.

[4] ARREBOLA J C, CABALLERO A, CRUZ M, et al. Crystallinity control of a nanostructured $\mathrm{LiNi}_{0.5} \mathrm{Mn}_{1.5} \mathrm{O}_{4}$ spinel via polymerassisted synthesis: a method for improving its rate capability and performance in $5 \mathrm{~V}$ lithium batteries. Adv. Funct. Mater., 2006, 16(14): 1904-1912.

[5] GOODENOUGH J B, PARK K S, The Li-ion rechargeable battery: a perspective. J. Am. Chem. Soc., 2013, 135(4): 1167-1176.

[6] RUHUL A, ILIAS B. Part I: Electronic and ionic transport properties of the ordered and disordered $\mathrm{LiNi}_{0.5} \mathrm{Mn}_{1.5} \mathrm{O}_{4}$ spinel cathode. $J$. Power Sources, 2017, 348: 311-317.

[7] LIU D, ZHU W, TROTTIER J, et al. Spinel materials for high-voltage cathodes in Li-ion batteries. RSC $A d v$., 2014, 4(1): 154-167.

[8] YOON T, PARK S, MUN J, et al. Failure mechanisms of $\mathrm{LiNi}_{0.5} \mathrm{Mn}_{1.5} \mathrm{O}_{4}$ electrode at elevated temperature. J. Power Sources, 2012, 215: 312-316.

[9] MANTHIRAM A, CHEMELEWSKI K, LEE E S. A perspective on the high-voltage $\mathrm{LiMn}_{1.5} \mathrm{Ni}_{0.5} \mathrm{O}_{4}$ spinel cathode for lithium-ion batteries. Energy Environ. Sci., 2014, 7(4): 1339-1350.

[10] CABANA J, CABANS C M, OMENYA F O, et al. Compositionstructure relationships in the Li-ion battery electrode material $\mathrm{LiNi}_{0.5} \mathrm{Mn}_{1.5} \mathrm{O}_{4}$. Chem. Mater., 2012, 24(15): 2952-2964.

[11] SONG J, SHIN D W, LU Y, et al. Role of oxygen vacancies on the performance of $\mathrm{Li}\left[\mathrm{Ni}_{0.5-x} \mathrm{Mn}_{1.5+\times}\right] \mathrm{O}_{4}(x=0,0.05$, and 0.08) spinel cathodes for lithium-ion batteries. Chem. Mater., 2012, 24(15): 3101-3109.

[12] MA X H, KANG B, CEDER G. High rate micron-sized ordered $\mathrm{LiNi}_{0.5} \mathrm{Mn}_{1.5} \mathrm{O}_{4}$. J. Electrochem. Soc., 2010, 157(8): A925-A931.

[13] ZHENG J M, XIAO J, YU X, et al. Enhanced $\mathrm{Li}^{+}$ion transport in $\mathrm{LiNi}_{0.5} \mathrm{Mn}_{1.5} \mathrm{O}_{4}$ through control of site disorder. Phys. Chem. Chem. Phys., 2012, 14(39): 13515-13521.

[14] WANG Y, CAO G. Developments in nanostructured cathode materials for high-performance lithium-ion batteries. Adv. Mater., 2008, 20(12): 2251-2269.

[15] XIAO J, CHEN X, SUSHKO P V, et al. High-performance $\mathrm{LiNi}_{0.5} \mathrm{Mn}_{1.5} \mathrm{O}_{4}$ spinel controlled by $\mathrm{Mn}^{3+}$ concentration and site disorder. Adv. Mater., 2012, 24(16): 2109-2116.

[16] ZHANG X, CHENG F, ZHANG K, et al. Facile polymer-assisted synthesis of $\mathrm{LiNi}_{0.5} \mathrm{Mn}_{1.5} \mathrm{O}_{4}$ with a hierarchical micro-nanostructure and high rate capability. RSC Adv., 2012, 2(13): 5669-5675.

[17] ZHENG J, XIAO J, YU X, et al. Enhanced $\mathrm{Li}^{+}$ion transport in $\mathrm{LiNi}_{0.5} \mathrm{Mn}_{1.5} \mathrm{O}_{4}$ through control of site disorder. Phys. Chem. Phys., 2012, 14(39): 13515-13521.

[18] KIM H, MYUNG S T, YOON C S, et al. Comparative study of $\mathrm{LiNi}_{0.5} \mathrm{Mn}_{1.5} \mathrm{O}_{4-\delta}$ and $\mathrm{LiNi}_{0.5} \mathrm{Mn}_{1.5} \mathrm{O}_{4}$ cathodes having two crystallographic structures: $\mathrm{Fd} 3 \mathrm{~m}$ and $\mathrm{P}_{3} 32$ cathodes. Chem. Mater., 2004, 16(5): 906-914.

[19] JO M R, KIM Y L, KIM Y, et al. Lithium-ion transport through a 
tailored disordered phase on the $\mathrm{LiNi}_{0.5} \mathrm{Mn}_{1.5} \mathrm{O}_{4}$ surface for high-power cathode materials. ChemSusChem, 2014, 7: 2248-2254.

[20] LEE J, DUPRE N, AVDEEV M, et al. Understanding the cation ordering transition in high-voltage spinel $\mathrm{LiNi}_{0.5} \mathrm{Mn}_{1.5} \mathrm{O}_{4}$ by doping Li instead of Ni. Sci. Rep., 2017, 7: 6728-6739.

[21] CHANG Z R, CHEN Z J, WU F, et al. Preparation of $\mathrm{Li}\left(\mathrm{Ni}_{1 / 3} \mathrm{Co}_{1 / 3} \mathrm{Mn}_{1 / 3}\right) \mathrm{O}_{2}$ by spherical $\mathrm{Ni}_{1 / 3} \mathrm{Mn}_{1 / 3} \mathrm{Co}_{1 / 3} \mathrm{OOH}$ at a low temperature. J. Power Sources, 2008, 185: 1408-1414.

[22] IDEMOTO Y, NARAI H, KOURA N. Crystal structure and cathode performance dependence on oxygen content of $\mathrm{LiMn}_{1.5} \mathrm{Ni}_{0.5} \mathrm{O}_{4}$ as a cathode material for secondary lithium batteries. J. Power Sources, 2003, 119-121: 125-129.

[23] BACON G E. Coherent neutron scattering amplitudes. Acta Crystallographica Section A, 1972, 28(4): 357-358.
[24] ARIYOSHI K, IWAKOSHI Y, NAKAYAMA N, et al. Topotactic two-phase reactions of $\mathrm{Li}\left[\mathrm{Ni}_{1 / 2} \mathrm{Mn}_{3 / 2}\right] \mathrm{O}_{4}\left(\mathrm{P}_{3} 32\right)$ in nonaqueous lithium cells. J. Electrochem. Soc., 2004, 151(2): A296-A303.

[25] OH S H, CHUNG K Y, JEON S H, et al. Structural and electrochemical investigations on the $\mathrm{LiNi}_{0.5-x} \mathrm{Mn}_{1.5-y} \mathrm{M}_{x+y} \mathrm{O}_{4}(\mathrm{M}=\mathrm{Cr}, \mathrm{Al}$, $\mathrm{Zr}$ ) compound for $5 \mathrm{~V}$ cathode material. J. Alloys. Compd., 2009, 469 (1/2): 244-250.

[26] TERADA Y, YASAKA K, NISHIKAWA F, et al. In situ XAFS analysis of $\mathrm{Li}(\mathrm{Mn}, \mathrm{M})_{2} \mathrm{O}_{4}(\mathrm{M}=\mathrm{Cr}, \mathrm{Co}, \mathrm{Ni}) 5 \mathrm{~V}$ cathode materials for lithium-ion secondary batteries. J. Solid State Chem., 2001, 156(2): 286-291.

[27] AMMUNDSEN B, ROZIERE J, ISLAM M S A. Atomistic simulation studies of lithium and proton insertion in spinel lithium manganese oxide. J. Phys. Chem. B, 1997, 101(41): 8155-8163. 